\title{
Machine Vision Techniques for Improve Rice Grain Quality Analyzing Process
}

\author{
G.M.K.B. Karunasena* \\ Department of Mechanical Engineering \\ The Open University of Sri Lanka
}

\author{
H.D.N.S. Priyankara \\ Department of Mechanical Engineering \\ The Open University of Sri Lanka
}

\author{
B.G.D.A. Madushanka \\ Department of Mechanical Engineering \\ The Open University of Sri Lanka
}

\begin{abstract}
Rice grain quality inspection is a major process in rice production. To provide quality and accurate results in rice grain analyzing it is important to analyze rice grains one by one in a testing sample. In the current situation, most of rice grain producers inspect rice grains manually without using any automated process. The major problem is the accuracy of testing results depends on human quality because manually processes include human errors. The manual inspection of rice grains is a very complicated and time-consuming process due to these reasons most of the inspector's effect by external factors such as fatigue, tension etc. In this research, we provide a time-efficient and low-cost solution for reducing above-mentioned limitations by developing software. It uses modern image processing to analyze rice grains one by one efficiently over the manual examination. The quality of rice samples can be determined with the help of colour, and geometric features such as area, maximum length, maximum width and aspect ratio. This analyzing system designed and developed for measure area, maximum length, maximum width and aspect ratio by using Java programming language, morphological and colour operations in computer vision and finally the accuracy of the system tested by comparing manually tested sample and results from the system. According to the results, it shows this system provides more than 85 percent accuracy with confirming this was a better solution.
\end{abstract}

Keywords:- Image Processing, Rice Grain Quality, Morphological Operations, Java For Image Processing, Rice Grading.

\section{INTRODUCTION}

Rice is the most famous food in many countries in the Asian region. It satisfies $21 \%$ of calorific demand of the entire human being. In Asia, around $90 \%$ of the rice produced and consumed [1]. Sri Lanka Ministry of Agriculture state that $37 \%$ of rice cultivation is occupying by the total cultivation of the country itself. Rice exportation is leading to the solution to global rice demand (approximately 1000 MT) by $1.95 \%$ annually. Peoples are practicing new technologies to increase the production, packaging and bagging of rice [2]. The categorization of rice grains in the rice industry makes attractive prices for rice grains from consumers for the farmers especially in Sri Lanka therefore there is a new requirement of image processing tools for the rice industry. Machine vision and image processing techniques are widely used in many disciplines such as biomedical, food, military etc. which reduce hardware cost. In present, many developers used different image processing techniques to quality inspection of rice grains. According to researchers, the rice grain shape decides by using area, length and width of rice grain. The classification of rice grain according to earlier researches is shows in the following tables. The rice grains can be classified according to their length as the classification shows in table I. Table II provides a classification based on the length breadth ratio, classified as slender, medium, bold and round, depending on the grains, which determines the shape of that grain. The given tables used for sort the rice grains according to their physical shape [3].

\begin{tabular}{|c|c|}
\hline Grain Size & Length rate $(\mathbf{m m})$ \\
\hline Extra-Long & $>7.5$ \\
\hline Long & $6.61-7.7$ \\
\hline Medium & $5.5-6.6$ \\
\hline Short & 5.5 or less \\
\hline
\end{tabular}

Table 1:- Classification based on length

\begin{tabular}{|c|c|}
\hline Grain Shape & L/W Ratio \\
\hline Slender & Over 3 \\
\hline Medium & $2.2-3$ \\
\hline Bold & $1.1-2$ \\
\hline Round & 1 or less \\
\hline
\end{tabular}

Table 2:- Classification based on L/W ratio

At present, rice grain length and width normally determined by an inspector by using a ruler or micrometer. The quality inspector manually measure length and width of grains in the testing sample by taking one by one, and al so it is hard and it takes considerable time. Expert professionals also carry out the investigation of the type of grain and its consistency parameters manually. This technique faces many drawbacks, mostly subject driven and influenced by human factors and working conditions. Human perception can easily affected by external factors such as eyesight and fatigue, work pressure and mental state caused by biases and working conditions such as climate and improper lighting, etc. That leads to inconsistent results, that rate of salvage clean up and recovery is limited, hence this manual practice thus affects the farmers. Due to the above mention reasons, this process require automation and develop imaging systems that can be helpful to identify rice grain images, rectify it \& then analyzed. This research provided software solution with image processing method for evaluating physical quality of rice grain. Through this method, we can improve the 
accuracy of inspection results and results can obtain minimum time. There are some unsolved problems remains in the use of image processing techniques for rice grading process such as unwanted lighting conditions from the environment and more filtration can cause deviation on results [3]. In this research, we use regulated light condition and uniform context for removing drawbacks mentioned above. The maximum grain length (major axis) and maximum width (minor axis) found by using morphological operations on each rice grain in sample and measure the L/W ratio. Then by applying grading formula, system grades rice grains in the given sample. The grading process done by according to rice grain standards mentioned in earlier researches. In addition, we are rating rice grains according to their region (area) in this research. Therefore first we calculate average area of pretested quality rice grain sample and calibrate the software an according to this average value we were detect low quality rice grins according to their area. Furthermore the theories and algorithms are used for proposed system approach explain in below.

\section{RELATED WORKS}

Different applications in the field of image processing was document in agriculture, biomedical engineering, food and drug industries, and many others. Image processing techniques to identify plants and background pixels in images of rice plants acquired in a field experiment developed. Rice is one of the world's main food crops, and is cultivated throughout the world. Rice is comparatively high in caloric value and rice protein has a good balance of the essential amino acids. Historically and now through planned breeding, each grain type is associated with specific milling, cooking and processing characteristics [3]. There are several varieties of each type of grain in commercial production and new ones are constantly being produce and released. Techniques developed to calculate the quality of the grain sample based on it; samples can be grade with respect to productivity and price. The sample standard has also proved its value in the breeding of cereals. The standard of the samples has also proved its value in cereal breeding. Herath, proposed Matlab solution for filter and classify four types of rice in a grain mixture. Rice sample represents the four varieties of rice grains (Red Basmati, White Basmati, Red Samba and White Samba) mix in together by using image processing [4]. Some researchers conclude, There are some different factors to determine the rice grain quality such as size, shape, colour, texture and to detect the given parameter, Several image processing techniques have been employed to make rice grading more effective such as the classification based on RGB, greyscale and intensity values from the image acquired [5]. The image acquisition process is the most important part because the noises of the input image directly affect the final results therefor some researchers used a black background for image acquisition process and they mentioned image processing performance can be improved due to black background [6]. J.S. Aulakh and V.K. Banga examined the pureness of the rice sample depends on a rice grain. The types of food grains and their consistency rapidly evaluated by human inspectors by visual inspection. According to the paper, human inspector's decision-making capabilities may be subject to external influence like fatigue, vengeance, bias etc. They provide image-processing solution to identify any broken grains mixed by using a desktop scanner [7]. According to the literature lots of research works done on grading different types of food-grains but there are less amount of researches conducted on rice grain grading. Therefore, we need to replace the manual rice grading process by using an automated process, therefore we identify the problem as design and implement of speed and cost effective method for rice grading by extracting the features of the grain such as width, length, area from the images obtained the grains by using digital image processing technique.

\section{METHODOLOGY}

This section mainly focused on, design and development of proposed rice grain analyzing software. In this system, the quality analyzing process done by according to physical properties of rice grains i.e. grain width, length and area using image-processing techniques. The parameters we used to rice grain grading:

Average length of rice sample.

Length of each rice grain.

$>$ Average $\mathrm{L} / \mathrm{W}$ ratio of rice sample.

$>$ Average $\mathrm{L} / \mathrm{W}$ ratio each rice grain.

$>$ Rice quality analysis according to area.

According to the above parameters, we developed methodology to rice grain grading by using some image processing techniques. The steps of proposed methodology is shows in Fig. 1.

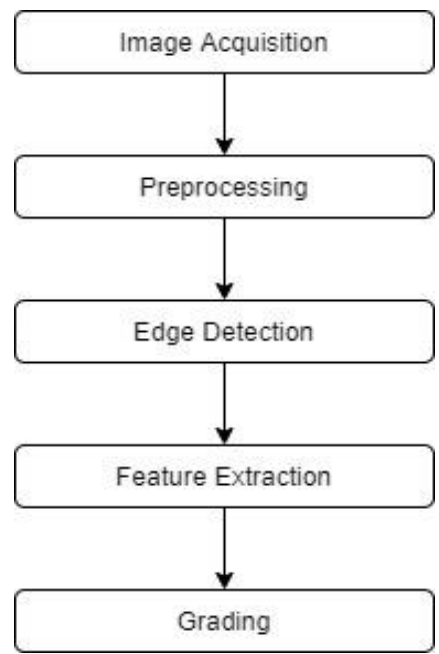

Fig 1:- Flow Diagram for Image Processing Algorithm

\section{A. Image Acquisition}

The image acquisition part is the most important part of the grain analyzing process because it directly affects the result of the process due to the image quality of rice grain samples. It is important to eliminate noises from the external environment to proper results. The image acquisition process generates a three-dimensional array according to red, green, blue values between 0-255. The input images 
captured using a USB colour camera with a $640 \times 480$ pixels resolution as shown in Fig. 3. The Fig. 2 shows the physical setup used for image acquisition process. This setup mainly integrated with front lighting system [8] for maintain continues lighting condition and remove unwanted lighting noises and the rice samples placed inside the light box.

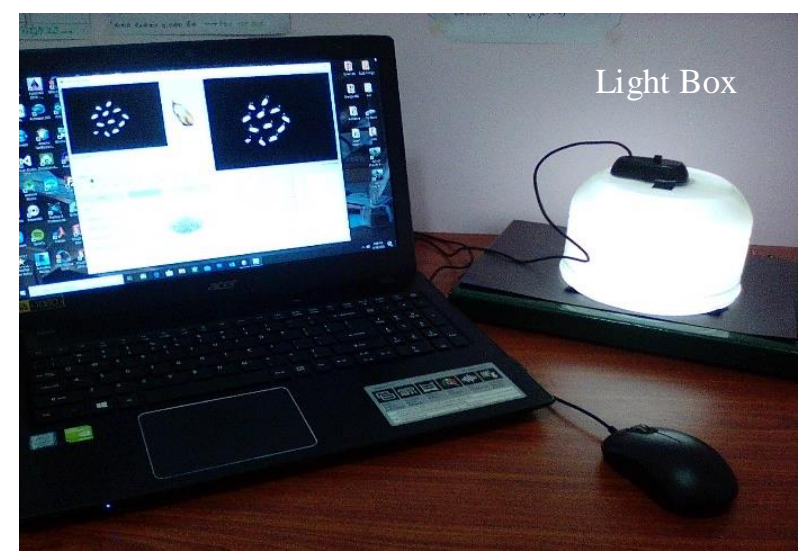

Fig 2:- Image Acquisition Setup

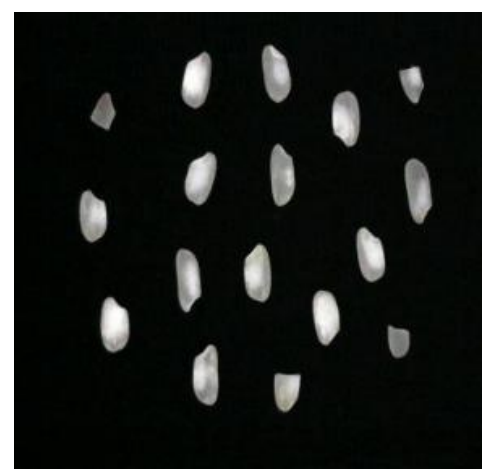

Fig 3:- Input Image

\section{B. Image Preprocessing-Gray scale image}

The captured image saved using the colour camera which saved in the three dimensional RGB (red, green, blue) colour space. Then the image will have to convert to greyscale. The greyscale images consist of pixels with an intensity range of pixel value between 0 and 255 , as shown in Fig.4. The greyscale conversion reduces the algorithm processing time and complexity of RGB to greyscale conversion. During the conversion, the pixel intensity differed depending on the different grain colours.

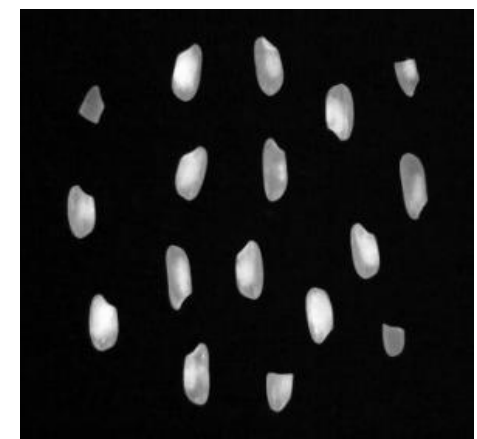

Fig 4:- Grey Scale Image

\section{Image Preprocessing-Binary image}

Then the greyscale image transformed into the binary image, as shown in Fig. 5. It contains only black (binary value 0 ) and white pixels (binary value 1 ). The binary image generates by performing the Threshold function on the greyscale image. Due to this thresholding, we can subtract rice grain areas from the main background for feature extraction.

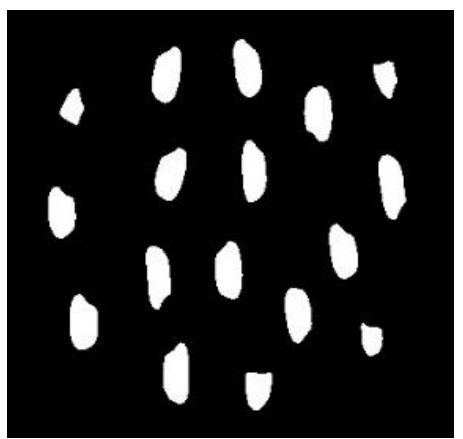

Fig 5:- Binary Image

Furthermore the areas of grains extract from background by identifying pixels that are consists value of binary value one.

\section{Edge Detection}

The edge detection algorithm used to find out the boundaries of rice grains in the binary converted image as shown in Fig. 5. In this research, we used border (contours) following algorithm [9]. In shape analysis, object detection and recognition processes the object contour is the main requirement because the contour indicates the object boundary. The detected contours are marked (green colour border) on sample image as shown in Fig. 6 . Next, we extract features of rice grains by sing these contours.

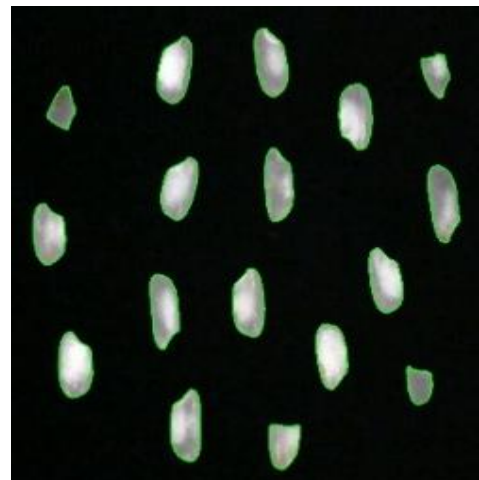

Fig 6:- Edges (contours) detected rice grains

\section{E. Image Preprocessing}

After finding contours, the algorithm finds the morphological properties of every rice grain using defined functions from the binary converted image. Morphological features included area, major axis length (L) and minor axis length (W) as shown in Fig. 7 extracted from images of rice grains. 


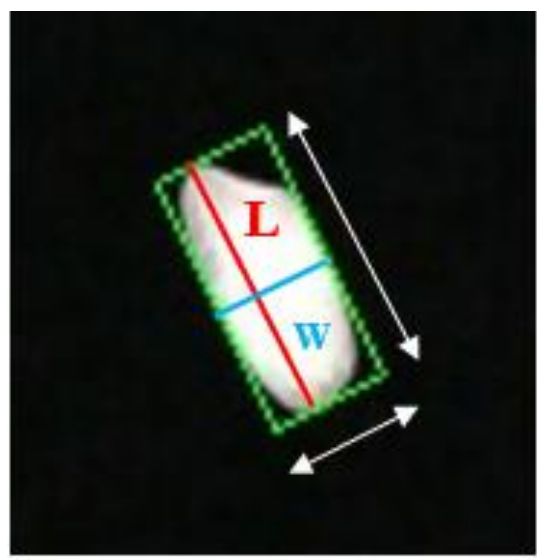

Fig 7:- Edge (contour) detected rice grain, $\mathrm{L}$ is the major axis length and $\mathrm{W}$ is minor axis length

\section{> Maximum Length (Major axis length)}

It was the distance between the endpoints of the longest line (L) as shown in Fig. 7 that drawn through the rice grain. The major axis length $(\mathrm{L})$ found by calculating the pixel distance between every combination of pixels in the grain contour.

\section{Maximum Width (Minor axis length)}

The minor axis (W) is the maximum distance between endpoints of the line as shown in Fig. 7 that drawn through the rice grain while maintaining perpendicularity with the major axis (L).

\section{Area}

This refers to the amount white of pixels in the region. The white pixel-counting algorithm used for calculate white pixels inside the rice grain area and calculate pixels on rice grain boundary $\left(\mathrm{mm}^{2} / \mathrm{pixel}\right)$. The individual pixel area was calibrate by using known $20 \times 20\left(\mathrm{~mm}^{2} /\right.$ pixel $)$ square area.

\section{GRAPHICAL USER INTERFACE}

The GUI (graphical user interface) is a computer software framework with interactive visual components. GUI shows objects conveying knowledge, and represents actions that the user may take. In this research, we used Java SE platform to develop this application so it can run any operating system such as Windows, Linux, etc. The developed GUI for the proposed method shows in Fig. 8. The visibility value (visibility slider shown in Fig. 8) use to set threshold value for generate binary image and this threshold value should set manually by user until the system identify rice grain border. In processed view, the green squares indicates accepted rice grains and red squares indicates rejected rice grains according to their $2 \mathrm{D}$ area. The all other parameters (count, L/W ratio, length, area) measured by the system indicates on GUI. Therefore, it reduce measuring time and user can easily get idea about rice sample.
ISSN No:-2456-2165

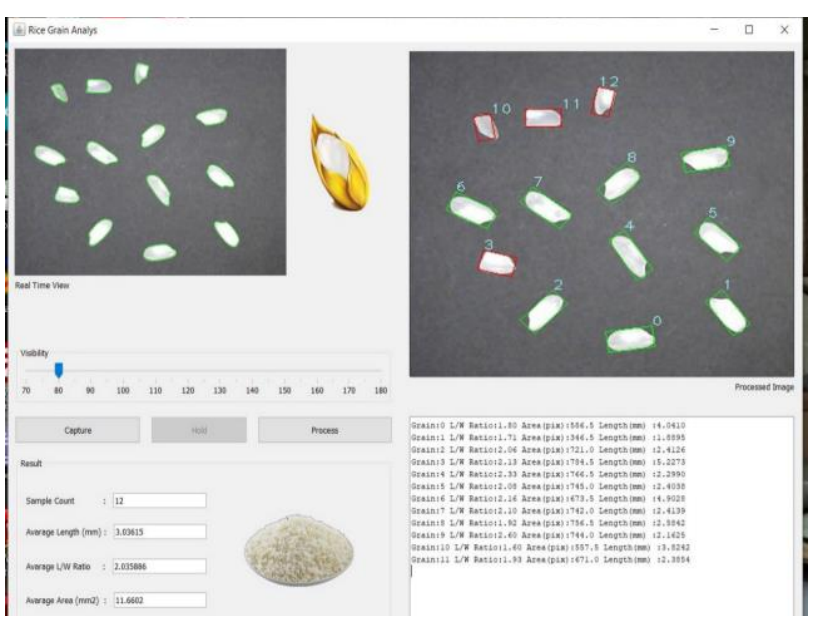

Fig 8:- Developed graphical user interface

\section{RESULTS AND DISCUSSION}

The numerical result of the proposed system presented in the following tables. The table I and table II represents the generated values of the proposed system. For the validation of the accuracy of the system, we tested and compared manually measured values and system measured values. The evaluation of the proposed system done by using four categories of rice grains, with total rice grain 40 in quantity. In the table $\mathbf{3}$, it presents four types of numerical results of the rice grain such as Extra-Long, Long, Medium and Short.

\begin{tabular}{|c|c|c|c|c|c|}
\hline Category & $\begin{array}{c}\text { Sample } \\
\text { Size }\end{array}$ & $\begin{array}{c}\text { Manual } \\
(\mathbf{m m})\end{array}$ & $\begin{array}{c}\text { System } \\
(\mathbf{m m})\end{array}$ & $\begin{array}{c}\text { Error } \\
(\boldsymbol{\%})\end{array}$ & $\begin{array}{c}\text { Accurate } \\
(\boldsymbol{\%})\end{array}$ \\
\hline $\begin{array}{c}\text { Extra- } \\
\text { Long }\end{array}$ & 10 & 7.7 & 7.58 & 12 & 88 \\
\hline Long & 10 & 6.9 & 6.77 & 13 & 87 \\
\hline Medium & 10 & 6 & 5.88 & 12 & 88 \\
\hline Short & 10 & 4.5 & 4.35 & 15 & 85 \\
\hline
\end{tabular}

Table 3:- Results of tested sample - Average length of rice grains

The results presented in table 3 shows average length measured by manual and developed system. It shows the system gives more than $85 \%$ of accurate value for grain length. In the following table 4 , it presents four types of numerical results of the rice grain such as Slender, Medium, Bold and Round.

\begin{tabular}{|c|c|c|c|c|c|}
\hline Category & $\begin{array}{c}\text { Sample } \\
\text { Size }\end{array}$ & Manual & System & $\begin{array}{c}\text { Error } \\
(\%)\end{array}$ & $\begin{array}{c}\text { Accurate } \\
(\%)\end{array}$ \\
\hline $\begin{array}{c}\text { Extra- } \\
\text { Long }\end{array}$ & 10 & 3.5 & 3.43 & 7 & 93 \\
\hline Long & 10 & 2.6 & 2.55 & 5 & 95 \\
\hline Medium & 10 & 1.8 & 1.75 & 5 & 95 \\
\hline Short & 10 & 0.9 & 0.84 & 6 & 94 \\
\hline
\end{tabular}

Table 4:- Results of tested sample - Average L/W ratio of rice grains 
The results presented in table III shows average L/W ratio measured by manual and developed system. It shows the system gives more than $90 \%$ of accurate value for grain $\mathrm{L} / \mathrm{W}$ ratio. These error differences show that the propose system is quite precise compared to the manual method of rice grain analyzing. According to numerical results generated from the proposed system, we can see the method used to proposed systems successful in recognition of lowquality rice grains and it is cost-effective as well. Furthermore, this software provides $2 \mathrm{D}$ area of the rice grains by counting pixels, the results presented in table $\mathrm{v}$ and table VI. Therefore, we can identify broken rice grains in grain sample by comparing area of each grain with average area value given by quality grain sample.

\begin{tabular}{|c|c|c|}
\hline Grain No. & Pixel Count & Area $\left(\mathbf{m m}^{\mathbf{2}}\right)$ \\
\hline 1 & 757 & 13.09 \\
\hline 2 & 785 & 13.57 \\
\hline 3 & 731 & 12.64 \\
\hline 4 & 795 & 13.74 \\
\hline
\end{tabular}

Table 5:- Results of tested sample - Area of full rice grains

\begin{tabular}{|c|c|c|}
\hline Grain No. & Pixel Count & Area $\left(\mathbf{m m}^{\mathbf{2}}\right)$ \\
\hline 1 & 331 & 5.72 \\
\hline 2 & 344 & 5.94 \\
\hline 3 & 390 & 6.74 \\
\hline 4 & 385 & 6.65 \\
\hline
\end{tabular}

Table 6:- Results of tested sample - Area of broken rice grains

\section{CONCLUTION}

In this research, we proposed a cost-effective and rapid technique for automating rice grading process. The proposed system includes a physical cover with a camera and front lighting system to capture rice grain samples and the proposed algorithm process the images of grain samples and extract the physical characteristics of grains in the sample the grade rice grains according to the predefined rice grain standards. The all proposed morphological operations included in the Java software to improve the useability of the system and feature extraction of the rice grin image sample. Then it provides results on the developed GUI in less time than manual inspection. According to the results, we can prove that the proposed system process rice grains efficiently with good accuracy, in a short period of time than manual inspection. Furthermore, this method provides a solution to measure 2D area of rice grains to identify broken grains..

\section{ACKNOWLEDGMENT}

The authors would like to acknowledge everyone how gave suggestions for this research and special thanks to The Open University of Sri Lanka for providing laboratory facilities for this research.

\section{REFERENCES}

[1]. R. Sharma, R. Khanna, Rice grain quality: Current developments and future prospects, IntechOpen; 2019.DOI: 10.5772/intechopen.89367

[2]. Sri Lanka Ministry of Agriculture. [Online]. Available:

https://doa.gov.lk/rrdi/index.php?option=com_sppage builder\&view=page \&id=42\&lang=en. [Accessed: 16Jun-2020]

[3]. N.A. Kuchekar and V.V Yerigeri, "Rice Grain Quality Grading Using Digital Image Processing Techniques", IOSR Journal of Electronics and Communication Engineering (IOSR-JECE) e-ISSN: 2278-2834, pISSN: 2278-8735.Volume 13, Issue 3, Ver. I (May. June. 2018), PP 84-88.

[4]. K. Herath, "IMAGE PROCESSING ANALYSIS FOR THE RICE GRAIN QUALITY IN SRI LANKA.," The Official E-Newsletter of the Institution of Engineers Sri Lanka, no. 33, Apr. 2017.

[5]. Guang-rong, Liu. "Rice color inspection based on image processing technique." 2010 International Conference on Advances in Energy Engineering (2010): 134-137.

[6]. Y.S.A. Naseem, Z. Ahmed, M. Faiz, M.U. Nisa, S.M.Z. Uddin and B. Khan, "An Economical Design of Automatic Rice Grading using Image Processing",International Journal of Recent Technology and Engineering (IJRTE) ISSN: $2277-$ 3878, Volume-8 Issue-2S9, September 2019

[7]. J.S. Aulakh and V.K. Banga, "GRADING OF RICE GRAINS BY IMAGE PROCESSING", International Journal of Engineering Research \& Technology (IJERT) Vol. 1 Issue 4, June - 2012 ISSN: 2278-0181

[8]. Bright field, front light illumination in machine vision - Opto Engineering Basics. 2020. Bright field, front light illumination in machine vision - Opto Engineering Basics. [ONLINE] Available at: https://www.opto-e.com/basics/bright-field-frontlight-illumination. [Accessed 12 June 2020].

[9]. Suzuki, Satoshi and K. Abe. "Topological structural analysis of digitized binary images by border following." Comput. Vis. Graph. Image Process. 30 (1985): 32-46. 